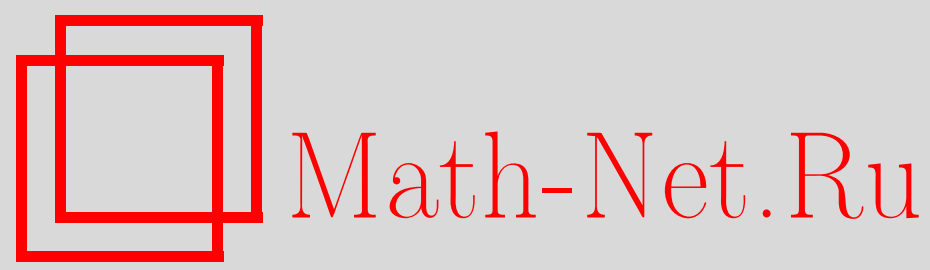

Е. А. Поляков, Об $R$-универсальных функциях, Матем. заметки, 2005, том 78, выпуск 2, 259-264

DOI: https://doi.org/10.4213/mzm2583

Использование Общероссийского математического портала Math-Net.Ru подразумевает, что вы прочитали и согласны с пользовательским соглашением http://www . mathnet.ru/rus/agreement

Параметры загрузки:

IP : 44.207 .124 .84

26 апреля 2023 г., 17:53:09

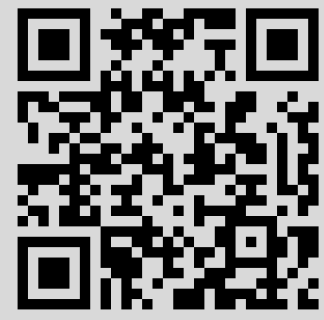


УДК 510.6

\section{ОБ $R$-УНИВЕРСАЛЬНЫХ ФУНКЦИЯХ}

\section{Е. А. Поляков}

Доказано, что тип $\rho$-сходства $R$-универсальной функции $(R \neq \varnothing, \mathbb{N})$ состоит из одного типа изоморфизма, но более чем из одного типа $\rho$-рекурсивного изоморфизма.

Библиография: 3 названия.

Как обычно, $\mathbb{N}$ - множество всех натуральных чисел $\{0,1,2, \ldots\}$. Если $A \subseteq \mathbb{N}$, то $\bar{A}=\mathbb{N} \backslash A$. Под функцией, если не оговорено противное, будем понимать одноместную частично рекурсивную функцию (ЧРФ). Обозначать ЧРФ будем греческими буквами: $\alpha, \beta, \ldots$, а рекурсивные функции - латинскими: $f, g, h, \ldots$ Пусть $\delta \alpha, \rho \alpha, \tau \alpha$ обозначают соответственно область определения, область значений, график функции $\alpha$; $\alpha^{a}=\{x \mid \alpha(x)=a\}-a$-уровень функции $\alpha ; \varphi_{x}-$ ЧРФ с гёделевьм номером $x$. Будем писать $\alpha(x)$, если значение $\alpha(x)$ определено, и $\alpha(x)$ 个 в противном случае. Через $|A|$ обозначим мощность множества $A$.

На множестве всех одноместных ЧР $\Phi$ определим пять отношений эквивалентности: сходство, $\rho$-сходство, изоморфизм, рекурсивньй изоморфизм и $\rho$-рекурсивный изоморфизм.

ОПРЕДЕЛЕНИЕ 1. ЧРФ $\alpha$ сходна с $\beta$ (обозначение: $\alpha \sim \beta$ ), если существуют рекурсивные перестановки $f$ и $g$ такие, что $\alpha=f \beta g$.

ОПРЕДЕЛЕНИЕ 2 . ЧРФ $\alpha \rho$-сходна с $\beta$ (обозначение: $\alpha \sim \rho \beta$ ), если существуют рекурсивные перестановки $f$ с условием $f(\rho \alpha)=\rho \alpha$ и $g$ такие, что $\alpha=f \beta g$.

ОПРЕДЕЛЕНИЕ 3 . ЧРФ $\alpha$ изоморфна $\beta$ (обозначение: $\alpha \approx \beta$ ), если существует рекурсивная перестановка $f$ такая, что $\alpha=\beta f$.

ОПРЕДЕЛЕНИЕ 4. ЧРФ $\alpha$ рекурсивно изоморфна $\beta$ (обозначение: $\alpha \equiv \beta$ ), если существует рекурсивная перестановка $f$ такая, что $\alpha=f^{-1} \beta f$.

ОПРЕДЕЛЕНИЕ 5 . ЧРФ $\alpha$-рекурсивно изоморфна $\beta$ (обозначение: $\alpha \equiv \rho \beta$ ), если существует рекурсивная перестановка $f$ с условием $f(\rho \alpha)=\rho \alpha$ такая, что $\alpha=f^{-1} \beta f$.

Очевидно,

$$
\alpha \approx \beta \Longrightarrow \alpha \sim_{\rho} \beta \Longrightarrow \alpha \sim \beta, \quad \alpha \equiv \rho \beta \Longrightarrow \alpha \equiv \beta, \quad \alpha \equiv_{\rho} \beta \Longrightarrow \alpha \sim_{\rho} \beta,
$$

для любых ЧРФ $\alpha, \beta$. Классы эквивалентных элементов будем назьвать соответственно типами сходства, $\rho$-сходства, изоморфизма, рекурсивного изоморфизма и $\rho$-рекурсивного изоморфизма. 
Если $\alpha, \beta-$ ЧР $\Phi$, то говорим, что $\alpha m$-сводима к $\beta$ (обозначение: $\alpha \leqslant m \beta$ ), если сушествует рекурсивная функция $f$ такая, что $\alpha=\beta f$.

ЧРФ $\psi$ назовем универсальной (m-полной), если для любой ЧРФ $\alpha$ имеет место $\alpha \leqslant_{m} \Psi$.

ОПРЕДЕЛЕНИЕ 6 . ЧРФ $\alpha$ назовем $R$-универсальной, если $R$ - рекурсивно перечислимое (РП) множество, $\rho \alpha=R$ и для любой ЧРФ $\beta \rho \beta \subseteq R \Longrightarrow \beta \leqslant{ }_{m} \alpha$.

Настоящая работа посвящена изучению свойств $R$-универсальных функций. Отметим несколько из полученных результатов:

$1)$ свойство функции быть $R$-универсальной инвариантно относительно $\rho$-сходства;

2 ) если $R \neq \varnothing, N$, то тип $\rho$-сходства $R$-универсальной функции состоит более, чем из одного типа $\rho$-рекурсивного изоморфизма;

3 ) если $|R| \geqslant 2$, то $R$-универсальная функция не имеет рекурсивных доопределений. Хорошо известно (см. [1]), что любые две $R$-универсальные функции изоморфны. С точностью до изоморфизма для произвольного РП множества $R R$-универсальной функцией будет

$$
\psi_{R}(x)= \begin{cases}\psi(x), & \text { если } \psi(x) \in R ; \\ \uparrow & \text { в противном случае }\end{cases}
$$

где $\psi$ - универсальная функция. Действительно, если $\alpha-$ ЧРФ и $\rho \alpha \subseteq R$, то $\alpha(x)=$ $\psi f(x)$ для некоторой рекурсивной функции $f$. Имеем $\alpha_{R}(x)=(\psi f)_{R}(x)$, откуда $\alpha(x)=$ $\psi_{R} f(x)$.

ПРЕДЛОЖЕНИЕ 1. а) Если $\gamma-R$-универсальная функиия $u g$-рекурсивная функиия с $\rho g=N$, то $\gamma g$ R-универсальна.

б) Пусть $f$-рекурсивная перестановка, $R$ - РП множество $u f(R)=R^{\prime}$. Тогда ЧРФ $\alpha-R$-универсальна в том и только том случае, когда $f \alpha R^{\prime}$-универсальна.

ДокАЗАТЕЛЬСТвО. а) Пусть $\alpha-$ ЧР $\Phi$ и $\rho \alpha \subseteq R$. Тогда $\alpha=\gamma f$ для некоторой рекурсивной функции $f$. Определим рекурсивную функцию $h(x)=\mu y(g(y)=f(x))$. Имеем $\alpha(x)=\gamma g h(x)$. Так как $\rho \gamma=R$, то $\rho(\gamma g) \subseteq R$. Пусть $r \in R$. Для некоторого числа $а \gamma(a)=r$. Так как $\rho g=N$, то $g(b)=a$ для некоторого $b$. Отсюда $\gamma g(b)=r$ и $r \in \rho(\gamma g)$. Значит, $R=\rho(\gamma g)$.

б) Необходимость. Пусть $\beta(x)-$ ЧРФ и $\rho \beta \subseteq R^{\prime}$. Тогда $\rho\left(f^{-1} \beta\right) \subseteq R$ и $f^{-1} \beta=\alpha g$ для некоторой рекурсивной функции $g$, откуда $\beta=f \alpha g$. Очевидно, что $\rho(f \alpha)=R^{\prime}$.

Достаточность очевидна.

Из предложения 1 вытекает, что свойство ЧРФ быть $R$-универсальной инвариантно относительно $\rho$-сходства. Используя изоморфизм $R$-универсальных функций, видим, что тип $\rho$-сходства $R$-универсальной функции состоит из одного типа изоморфизма. В [2] ЧР $\Phi$, тип $\rho$-сходства которых состоит из одного типа изоморфизма, названы $F_{\rho}$-функииям $u$; значит, $R$-универсальные функции будут $F_{\rho}$-функциями.

ПРЕДЛОЖЕНИЕ 2. Если $\alpha \quad R$-универсальна, $\beta$ - ЧРФ, $\rho \beta \subseteq R, \delta \beta \subseteq R$, то $\beta \alpha$ $\rho \beta$-универсальна. 
ДокАЗАТЕЛЬСтво. Очевидно, $\rho(\beta \alpha)=\rho \beta$. Пусть $\gamma-$ ЧР $\Phi$ и $\rho \gamma \subseteq \rho \beta$. Определим функцию $\gamma_{1}$. По данному $n$ находим $\gamma(n)$. Если $\gamma(n) \uparrow$, то и $\gamma_{1}(n) \uparrow$. А если $\gamma(n)=r \in R$, то перечисляя $\delta \beta$, ищем $k$ такое, что $\beta(k)=r$. Первое из таких найденных $k$ берем в качестве $\gamma_{1}(n)$. В силу тезиса Чёрча $\gamma_{1}-$ ЧР $\Phi ; \rho \gamma_{1} \subseteq R, \gamma_{1}=\alpha f$ для некоторой рекурсивной функции $f$, откуда $\beta \gamma_{1}=\beta \alpha f$ или $\gamma=\beta \alpha f$.

Точка $x=a$ назьвается неподвижной точкой функции $\alpha$, если $\alpha(a)=a$.

ПРЕДЛОЖЕНИЕ 3. Если $R$ - простое или ко-конечное множество, то $R$-универсальная функиия имеет креативное множсество неподвижных точек.

ДокаЗАтЕльСтво. Рассмотрим универсальную функцию $\psi$ и $R$-универсальную $\psi_{R}$. Пусть $I, I_{R}-$ множества неподвижных точек у функций $\psi$ и $\psi_{R}$ соответственно. Очевидно, что $I \cap R=I_{R}$. Деккер показал [3], что пересечение креативного и простого множеств является креативньг множеством. Значит, $I_{R}-$ креативное множество. Пусть $\alpha(x)-R$-универсальная функция. Тогда $\alpha(x)=\psi_{R} h(x)$, где $h$ - рекурсивная перестановка. Но $\alpha(x)=(\psi h)_{R}(x)$, где $\psi h$ - универсальная функция.

ПРЕДЛОЖЕНИЕ 4. Если $R$ - РП множество, не являющееся ни ко-конечным, ни простым, то существует $R$-универсальная функиия, не имеющая неподвихных точек.

ДокАЗАТЕЛЬСтво. Пусть $A$ - бесконечное РП подмножество $\bar{R}$. Оно содержит креативное множество $B$ (см. [3, упр. 7-47]). Пусть $f$-рекурсивная перестановка такая, что $f(B)=\delta \psi$, где $\psi$ - универсальная функция, откуда $f(\bar{B})=\overline{\delta \psi}$. Тогда $\psi^{\prime}=\psi f$ - универсальная функция и $R \subseteq \overline{\delta \psi^{\prime}}$. Рассмотрим $R$-универсальную функцию $\psi_{R}^{\prime}(x)$; она не имеет неподвижных точек. Действительно, если бы $\psi_{R}^{\prime}(a)=a$, то $\psi^{\prime}(a) \downarrow$ и $\psi^{\prime}(a)=a \in R$. Противоречие.

ПРЕДЛОЖЕНИЕ 5. Пусть $R$ - бесконечное РП множество, не являющееся ни простым, ни ко-конечным. Тогда для каждого натурального числа $n \geqslant 1$ существует $R$-универсальная функиия, имеющая в точности п неподвижных точек.

ДокАЗАТЕльство. Пусть $\alpha-R$-универсальная функция, не имеющая неподвижных точек. Выберем различные числа $a_{1}, \ldots, a_{n} \in R$. Некоторые из них могут принадлежать $\delta \alpha$. Пусть $a_{i_{1}}, \ldots, a_{i_{K}} \in \delta \alpha$. Выберем $c_{1}, \ldots, c_{n}$ так, чтобы

$$
\begin{gathered}
\alpha\left(c_{i}\right)=a_{i}, \quad\left\{a_{1}, \ldots, a_{n}\right\} \cap\left\{c_{1}, \ldots, c_{n}\right\}=\varnothing, \quad i=1, \ldots, n, \\
\alpha\left(a_{i_{j}}\right) \neq c_{i_{j}}, \quad j=1, \ldots, k .
\end{gathered}
$$

Определим рекурсивную перестановку

$$
f(x)= \begin{cases}c_{i}, & \text { если } x=a_{i} ; \\ a_{i}, & \text { если } x=c_{i} ; \\ x, & \text { если } x \neq a_{i}, c_{i} .\end{cases}
$$

Функция $\beta(x)=\alpha f(x)$ будет $R$-универсальной, имеющей в точности $n$ неподвижных точек. Действительно,

$$
\beta\left(a_{i}\right)=\alpha f\left(a_{i}\right)=\alpha\left(c_{i}\right)=a_{i}, \quad i=1, \ldots, n .
$$

Рассмотрим $\beta\left(c_{i}\right)=\alpha f\left(c_{i}\right)=\alpha\left(a_{i}\right)$; если $\alpha\left(a_{i}\right) \downarrow$, то $\alpha\left(a_{i}\right) \neq c_{i}$. 
ЗАмЕчАниЕ. Если $R$ - конечное множество и $|R|=n \geqslant 1$, то для каждого $1 \leqslant k \leqslant n$ существует $R$-универсальная функция, имеющая в точности $k$ неподвижных точек. Доказательство аналогично доказательству предложения 5.

ПРЕДЛОЖЕНИЕ 6. Для Каждого бесконечного РП множества $R$ существует $R$-универсальная функиия, имеющая бесконечное множество неподвижных точек.

ДокАЗАТЕЛЬСтво. Пусть $\alpha-R$-универсальная функция и $R$ - бесконечное РП множество. Построим бесконечные рекурсивные множества $A=\left\{a_{0}<a_{1}<\cdots\right\}$ и $B=$ $\left\{b_{0}<b_{1}<b_{2}<\cdots\right\}$ такие, что

$$
A \cup B=\left\{a_{0}<b_{0}<a_{1}<b_{1}<a_{2}<b_{2}<\cdots\right\}, \quad A \subseteq R, \quad B \subseteq \delta \alpha, \quad \alpha\left(b_{i}\right)=a_{i}
$$

для всех $i \in N$. Определим рекурсивную перестановку следующим образом:

$$
g(x)= \begin{cases}b_{i}, & \text { если } x=a_{i} ; \\ a_{i}, & \text { если } x=b_{i} ; \\ x, & \text { если } x \notin A \cup B .\end{cases}
$$

Положим $\beta(x)=\alpha g(x)$. Функция $\beta$ является $R$-универсальной и имеет бесконечное множество неподвижных точек. Действительно, $\beta\left(a_{i}\right)=\alpha g\left(a_{i}\right)=\alpha\left(b_{i}\right)=a_{i}$.

ПРЕДЛОЖЕНИЕ 7. График непустой $R$-универсальной функиии креативен.

ДокАЗАТЕЛьСТво. Пусть $\gamma-R$-универсальная функция, $a \in R$. Определим ЧРФ следующим образом:

$$
\alpha(x)= \begin{cases}a, & \text { если } x \in K ; \\ \uparrow, & \text { если } x \notin K,\end{cases}
$$

где $K$-какое-нибудь креативное множество. Тогда $\alpha(x)=\gamma f(x)$ для некоторой рекурсивной функции $f$. Имеем

$$
x \in K \Longleftrightarrow \alpha(x)=a \Longleftrightarrow \gamma f(x)=a \Longleftrightarrow\langle f(x), a\rangle \in \tau \gamma
$$

откуда $K \leqslant m$. Мы отождествляем пару чисел $(x, y)$ с ее канторовским номером $\langle x, y\rangle$.

ЗАмЕчАниЕ. Нетрудно доказать, что если график ЧР $\Phi \gamma$ креативен, то и $\delta \gamma$ креативна.

ЛЕммА. Пусть $\alpha-$ ЧРФ. Тогда свойство $\delta \alpha \subseteq \rho \alpha$ инвариантно относительно рекурсивного изоморфизма.

ДокАЗАТЕЛЬСТво. Имеем

$$
\delta \alpha \subseteq \rho \alpha \Longleftrightarrow \forall x(\alpha(x) \downarrow \Longrightarrow \exists y(\alpha(y)=x))) .
$$

Пусть $f$ - произвольная рекурсивная перестановка и

$$
f^{-1} \alpha f(x) \downarrow \Longrightarrow \alpha(f(x)) \downarrow \Longrightarrow \exists y(\alpha(y)=f(x)) .
$$

Получаем $f^{-1} \alpha f(z)=x$ при $z=f^{-1}(y)$. 
ПРЕДЛОЖЕНИЕ 8. Если $R \neq \varnothing, N$, то тип $\rho$-сходства $R$-универсальной функции состоит более, чем из одного типа $\rho$-рекурсивного изоморфизма.

ДокАЗАТЕЛЬСтво. Пусть $\gamma-R$-универсальная функция. Рассмотрим сначала случай, когда $R$ просто или ко-конечно и $R \neq \mathbb{N}$. Так как $A=\delta \gamma$ креативное, то и $A \cap \rho \gamma=B$ креативное. Существует рекурсивная перестановка $f$ такая, что $f(B)=A$. Определим $\gamma^{\prime}=\gamma f ; \gamma^{\prime}-R$-универсальная функция и $\delta \gamma^{\prime} \subseteq \rho \gamma^{\prime}$. Так как $R \neq \mathbb{N}$, существует $a \notin R$. Возьмем число $b$ такое, что $\gamma(b) \downarrow$ и $a \neq b$. Определим рекурсивную перестановку

$$
g(x)= \begin{cases}a, & \text { если } x=b ; \\ b, & \text { если } x=a ; \\ x, & \text { если } x \neq a, b,\end{cases}
$$

и положим $\gamma^{\prime \prime}(x)=\gamma g(x)$. Тогда $\gamma^{\prime \prime}-R$-универсальная функция, для которой $\gamma^{\prime \prime}(a) \downarrow$, но $a \notin \rho \gamma^{\prime \prime}$. В силу леммы $\gamma^{\prime}$ не может быть $\rho$-рекурсивно изоморфна $\gamma^{\prime \prime}$.

В случае, когда $R$ - бесконечное множество, не являющееся ни простьм, ни ко-конечным, тип $\rho$-сходства функции $\gamma$ распадается на бесконечное множество типов $\rho$-рекурсивного изоморфизма. В случае непустого конечного $R$ утверждение следует из предложения 4 и замечания после предложения 5.

ПРЕДЛОЖЕНИЕ 9. Если $|R| \geqslant 2$, то R-универсальная функиия не имеет рекурсивных доопределений.

ДокАЗАТЕЛЬСТво. Сначала покажем, что функция $\gamma(x)=\varphi(x)$ является универсальной. Очевидно, $\rho \gamma=N$. Пусть $\beta-$ ЧР $\Phi ; \beta=\gamma \alpha$ для некоторой ЧР $\alpha$. По $s$ - $m$ - $n$-теореме $\gamma \alpha(x)=\gamma^{\prime}(x, y)=\varphi_{s(x)}(y)$ для некоторой рекурсивной функции $s$. Имеем $\beta(x)=\gamma \alpha(x)=\gamma S(x)$, откуда $\beta \leqslant m \quad \gamma$. В силу предложения 2 функция $\psi(x)=$ $\overline{\operatorname{Sg}} \varphi_{x}(x)\{0,1\}$-универсальна, где

$$
\overline{\operatorname{Sg}} x=\left\{\begin{array}{l}
1, \text { если } x=0 ; \\
0, \text { если } x>0 .
\end{array}\right.
$$

Функция $\psi$ не имеет рекурсивных доопределений. Предположим, что $\psi$ имеет рекурсивное доопределение $f$. Тогда $f=\varphi_{a}$ для некоторого числа $a$ и, значит, $\varphi_{a}(a) \downarrow$. Имеем $\varphi_{a}(a)=\overline{\operatorname{Sg}} \varphi_{a}(a)$, что невозможно. Пусть $\eta-$ ЧР $\Phi, g$ и $f$ - рекурсивные перестановки. Нетрудно видеть, что $\eta$ имеет рекурсивное доопределение тогда и только тогда, когда $f \eta g$ имеет рекурсивное доопределение. Пусть $a, b$ - два различных числа. Построим $\{a, b\}$-универсальную функцию, не имеющую рекурсивных доопределений. Рассмотрим сначала случай, когда $1<a<b$. Определим рекурсивную перестановку

$$
h(x)=\left\{\begin{array}{l}
a, \text { если } x=0 ; \\
b, \text { если } x=1 ; \\
0, \text { если } x=a ; \\
1, \text { если } x=b ; \\
x, \text { если } x \neq 0,1, a, b .
\end{array}\right.
$$


Функция $h \psi$ будет искомой. Остальные случаи рассматриваются аналогично. Пусть, наконец, $\varkappa$ - произвольная $R$-универсальная функция, у которой $|R| \geqslant 2$. Выберем различные числа $a, b \in R$ и определим $\{a, b\}$-универсальную функцию следующим обра30м:

$$
\varkappa^{\prime}(x)= \begin{cases}\varkappa(x), & \text { если } \varkappa(x) \in\{a, b\} ; \\ \uparrow & \text { в противном случае. }\end{cases}
$$

Если бы $\varkappa$ имела рекурсивное доопределение, то и $\varkappa^{\prime}$ имела бы рекурсивное доопределение, что не так.

\section{СПИСОК ЦИТИРОВАННОЙ ЛИТЕРАТУРЫ}

[1] Ершов Ю. Л. Теория нумераций. М.: Наука, 1977.

[2] Поляков Е. А., Перов А. Е. F $\rho$-функции // Матем. заметки. 2003. Т. 74. № 4. С. 559-563.

[3] Роджерс X. Теория рекурсивных функций и эффективная выгислимость. М.: Мир, 1972.

Шуйский государственный педагогический университет

Поступило 20.02 .2004 